\title{
External Concurrent Occipital and Trigeminal Neurostimulation Relieves Migraine Headache: A Prospective, Randomized, Double-Blind, Sham- Controlled Trial
}

Oved Daniel ( $\sim$ danieloved@gmail.com )

Headache and Facial Pain Clinic Ramat-Aviv Medical Center

\section{Stewart J. Tepper}

Geisel School of Medicine at Dartmouth Hanover

Lisa Deutsch

BioStats Statistical Consulting, Ltd

Roni Sharon

Tel-Aviv University Sackler School of Medicine

\section{Research Article}

Keywords: peripheral nerve stimulation, trigeminal nerve, occipital nerve, neurostimulation, brain stimulation, neuromodulation, migraine, headache

Posted Date: February 24th, 2022

DOI: https://doi.org/10.21203/rs.3.rs-1385396/v1

License: (a) (i) This work is licensed under a Creative Commons Attribution 4.0 International License.

Read Full License 


\section{Abstract}

Background: Combined peripheral occipital and trigeminal neurostimulation is clinically proven to alleviate migraine symptoms, however, it was previously possible only via high-risk surgical procedures. Neuromodulation using currently available peripheral nerve stimulation devices stimulates only one nerve. The objective of this prospective, randomized, double-blind, sham-controlled trial was to evaluate the efficacy, safety and tolerability of a novel external concurrent occipital and trigeminal neurostimulation (eCOT-NS) device designed as a self-administered home treatment for migraine (Relivion ${ }^{\circledR}$ MG, Neurolief, Ltd; Netanya, Israel).

Methods: Episodic and chronic migraine subjects $(N=55)$ were randomized to receive active $(n=27)$ or sham $(n=28)$ treatment. Each subject received an eCOT-NS device and performed a $60 \pm 20$-minute treatment at home within 45 minutes of migraine episode onset. The primary endpoint was the change in mean baseline pain intensity based on VAS pain scores 1 hour after treatment initiation, defined as relative (percent) change. Treatment outcomes assessed at 1, 2 and 24 hours post-treatment initiation were pain-reduction, and proportion of pain-free subjects and treatment responders, defined as $\geq 50 \%$ pain reduction. Categorical pain ratings (none, mild, moderate, and severe pain) were also analyzed.

Results: Active stimulation was significantly more effective than sham stimulation for decreasing pain intensity at 1 hour (53\% vs.10\%), 2 hours (52\% vs. $17 \%$ ) and 24 -hours (71\% vs. 34\%). Pain-free ratings were greater for the active treatment arm at 1 hour $(29.2 \%$ vs. $16 \%), 2$ hours $(41.7 \%$ vs. $20 \%)$ and 24 hours $(65.2 \%$ vs. $40 \%)$. Subjects with moderate or severe migraine baseline pain who were pain-free at 2 hours was significantly greater among subjects receiving active treatment (43\% vs. $10.5 \%)$. The responder rate was significantly higher among the active treatment group at 1 hour $(67 \% v s .20 \%), 2$ hours $(66.7 \%$ vs. $32 \%$,) and 24 hours (78.3\% vs. 48\%). Overall headache-relief was significantly higher in the active treatment group at 1 hour ( $67 \%$ vs. $26 \%$ ) and 2 hours ( $76 \%$ vs. $31.6 \%)$. Mild adverse events, reported by a minority of subjects, resolved spontaneously.

Conclusions: eCOT-NS provides superior clinically meaningful relief and freedom of migraine pain compared to sham, offering a highly effective and safe non-pharmacological treatment for acute migraine.

Trial registration: ClinicalTrials.gov Identifier NCT03398668.

\section{Background}

Migraine is an extraordinarily prevalent neurological disease, affecting 39 million men, women and children in the U.S. and 1 billion people worldwide (1) with a much greater prevalence among women (2). Migraine patients experience disabling symptoms that typically consist of moderate-to-severe headache pain lasting 4 to 72 hours, nausea with or without vomiting, phonophobia and photophobia (3). Patients with migraine are often o treated with first-line therapy such as simple or combination analgesics that have variable onset and duration (4). The triptans are effective first-line treatment for moderate to severe 
migraine (4). Unfortunately, the safety and tolerability profile of these medications includes numerous potential adverse events (5), affecting approximately $50 \%$ of treated patients. Furthermore, triptans are contraindicated in patients with vascular disease (6) and have been associated with the development of headache due to medication overuse (7). Thus, there remains a significant unmet need for treating migraine pain.

Peripheral nerve stimulation (PNS) is a clinically established medical technology for brain neuromodulation using invasively implanted electrodes or non-invasive targeted delivery of electrical current. Surgically implanted PNS electrodes for treating migraine, such as occipital nerve stimulation, significantly attenuated chronic migraine in several clinical trials (8-13) but remains a costly procedure with a high rate of complications $(11,14)$. Consequently, these procedures are reserved for more severe, intractable cases of chronic migraine.

An alternative to implanted devices is the application of non-invasive, transcutaneous PNS. In shamcontrolled studies, single-channel stimulation of the trigeminal nerve is similar to other drug and non-drug treatments for migraine $(15,16)$. Similarly, supraorbital stimulation significantly reduces migraine pain compared to sham treatment (16). Among 2,313 patients treated with transcutaneous stimulation of the supraorbital branch of the trigeminal nerve, there were no reports of serious adverse events (17). A major shortcoming of these studies is that stimulation was limited to only the first division of the trigeminal nerve despite the reported efficacy of occipital nerve stimulation (18).

Concurrent invasive stimulation of both trigeminal and occipital nerves has been proposed as a more effective approach to minimize holo-cephalic pain due to migraine (19). Subsequently published clinical data support the hypothesis that invasive synchronous PNS of the occipital and supraorbital nerves may provide better outcomes compared with stimulation of the occipital nerve alone $(20,21)$. Using a response rate defined as $\geq 50 \%$ decrease in pain severity, subjects with intractable head-wide pain who were treated with implanted occipital and trigeminal nerve PNS achieved $70 \%(20)$ to over $90 \%$ improvement $(19,21)$. By comparison, occipital nerve stimulation alone only achieved an approximately $40 \%$ response rate $(9,10,13)$. Taken together, these reports suggest the possible superiority of bi-focal PNS over uni-focal PNS (20-24), however, these studies include high complication rates which emphasizes the need for similar but noninvasive approaches.

A means for delivering noninvasive, external concurrent occipital and trigeminal neurostimulation (eCOTNS) has been developed. The trigeminal nerve branches are easy to access externally, but transferring an electric current through the hair covering the occipital area required implanted (9-13) and percutaneous (25) nerve stimulators to achieve occipital nerve stimulation was technologically challenging. However, topographic analysis of occipital nerve branches challenged this assumption. As occipital nerve branches are located superficially $(26,27)$, accurate placement of stimulating electrodes can effectively and noninvasively stimulate the occipital nerve. Using this method, eCOT-NS may provide the same beneficial effects of invasive procedures without the high cost and risks associated with surgically implanted stimulation $(10,11)$, and while also avoiding the adverse events associated with medications. This is the 
rationale underlying the introduction of Relivion ${ }^{\circledR M G}$ (Neurolief, Ltd; Netanya, Israel), a US Food and Drug Administration-cleared eCOT-NS device for home use which includes six integrated electrodes that deliver mild electrical stimulation to the target nerves.

We hypothesized that the self-administered stimulation protocol using the Relivion ${ }^{\circledR} M G$ device can provide a safe, effective, and fast-acting acute reduction of migraine-related pain. The primary objective of this randomized, sham-controlled study was to assess changes in pain intensity following treatment.

\section{Materials And Methods}

This prospective randomized, double-blind, parallel-group, sham-controlled trial was conducted at a single site (Headache and Facial Pain Unit, Laniado Medical Center; Netanya, Israel). The study was conducted in accordance with principles of the Declaration of Helsinki (28), Good Clinical Practices, (ClinicalTrials.gov Identifier NCT03398668) and approved by the Institution Ethics Committee. All subjects provided written informed consent prior to participating in any study-related activities.

\section{Study Population}

Subjects eligible for inclusion were 18 to 65 years old with a history of episodic or chronic migraine, with or without aura, with no complication (i.e., hemiplegic migraine, basilar-type migraine, ophthalmoplegic migraine, migrainous infarction), who met the International Classification of Headache Disorders (ICHD-3 beta Section 1, Diagnostic Criteria for Migraine) (29). Enrolled subjects expressed their willingness to follow study requirements and were capable of following the study protocol including being able to selfoperate the stimulation system at home.

Reasons for exclusion from study participation were treatment with a neurotoxin or supraorbital or occipital nerve blocks in the planned treatment area during the previous 4 months; history of medication overuse headaches; prior cerebrovascular event, or neurosurgical interventions; history or current drug abuse or alcoholism; brain or facial trauma during the previous 3 months; skin lesions or inflammation in the location of the stimulating electrodes; diagnosis of epilepsy, personality or somatoform disorder; current opioid or cannabis use; implanted metal or electrical devices in the head (not including dental implants); implanted neurostimulators, surgical clips (above the shoulder line) or any medical pumps; pregnancy, nursing or planned pregnancy; unwillingness to use a medically acceptable method of contraception; participation in a clinical study within the previous 3 months; prior experience with the Relivion $\circledast$ MG device; or a head circumference less than 51 centimeters or greater than 60 centimeters.

\section{The Relivion®MG System}

Relivion ${ }^{\circledR} M G$ is a noninvasive system for external concurrent occipital and trigeminal neurostimulation (eCOT-NS). It is U.S. FDA-cleared and CE-approved for the acute treatment of migraine. A similar device has been developed for the treatment of major depression (Relivion®DP) (30). 
The eCOT-NS device is an ergonomic headset designed to externally stimulate superficial nerves in the head region. The novel design enables accurate and repeatable positioning of the embedded electrodes over the target nerves. The headset integrates three pairs of output electrodes, which contact the scalp at the forehead (two pairs) and occiput (one pair). The frontal electrodes stimulate the trigeminal supraorbital and supratrochlear nerve branches, and the posterior electrodes bilaterally stimulate the greater occipital nerve branches (Fig. 1). The device is adjustable to fit scalp anatomic variations and ensure the six electrodes are accurately positioned over the underlying nerves each time the headset is worn. The device incorporates a simple on-board interface which allows the user to activate/deactivate the device and to adjust the stimulation intensity. It provides visual and auditory indications such as if the device is active and its battery status.

A technical log is produced by the system after each use enabling full quantification of the treatment course. To enable remote treatment monitoring, the Relivion ${ }^{\circledR} M G$ system is connected to a custom mobile application, used by the patient, designed to continuously record data and securely upload it to a cloud database. Treatment reports and metrices are available to the investigator over a web-based physician interface (Fig. 1).

The following parameters were used for active stimulation: symmetrical biphasic waveform, phase width 400 microseconds, pulse frequency $80 \mathrm{~Hz}$, trigeminal stimulation intensity up to $6 \mathrm{~mA}$, occipital stimulation intensity up to $12 \mathrm{~mA}$. The following parameters were used for sham stimulation: symmetrical biphasic waveform, phase width 100 microseconds, pulse frequency $0.33 \mathrm{~Hz}$, trigeminal stimulation intensity up to $5 \mathrm{~mA}$, occipital stimulation intensity up to $7 \mathrm{~mA}$.

\section{Study Design}

Following eligibility and health screening, enrolled subjects were randomized 1:1 in double-blind manner, stratified by gender, to receive either active stimulation $(n=27)$, or sham (placebo) stimulation $(n=28)$ with a similar-appearing device (Fig. 2). Subjects were then trained on use of the eCOT-NS device and received an electronic or hard copy diary. Subjects were instructed to practice using the device and diary in their home environment twice, not during a migraine episode, for a duration of 20-60 minutes. Device logs were downloaded by the research clinician to ensure completion of at least one successful 20-60 home self-practice session.

Subjects were instructed to initiate treatment within $45 \pm 15$ minutes of migraine headache onset and not to use analgesics within 4 hours before treatment. Subjects used the personal electronic or hard copy diary to record pain intensities, use of analgesics, and adverse events (AEs). A baseline $\left(t_{0}\right)$ outcome measure of perceived pain intensity, recorded on a visual analog scale (VAS), was documented in the study diary prior to treatment initiation, and after treatment was initiated. Treatments with a stimulation time of more than 30 minutes were counted as a completed treatment. The subject then recorded the pain VAS score again at 1 hour ( $t_{1}$, end of treatment), 2 hours $\left(t_{2}\right)$ and 24 hours $\left(t_{3}\right)$ after treatment initiation. If necessary, rescue drugs were permitted 2 hours after treatment initiation. 
Subjects documented AEs and medication use in the study diaries. Upon completion of one treatment of a migraine headache, subjects returned the device to the clinic. Device logs were downloaded, reviewed, and documented to ascertain that the subject completed the treatment successfully. A successful treatment was defined as a recorded treatment with total stimulation time of more than 30 minutes and pass status (a minimum of $2 \mathrm{~mA}$ stimulation intensity) in the current device log. If the treatment was not successful, the subject was withdrawn from the analysis. AEs and concomitant medication change were documented.

Precautions were taken to avoid compromising subject blinding, including concealed allocation, use of an identical sham and active devices, and the same treatment protocol in the preliminary test stimulation and treatment sessions. Additionally, sham stimulation was set to a level well above sensory threshold to further enhance subject blinding.

\section{Outcome Measures}

Pain intensity was recorded prior to treatment and 1,2 and 24 hours after treatment initiation. The primary endpoint was the change in mean baseline pain intensity based on VAS pain scores 1 hour after treatment initiation and defined as relative (percent) change. The change in VAS score for each subject was calculated using the formula:

Relative Change $(\%)=$\begin{tabular}{l|} 
Post-treatment - Pre-treatment \\
Pre-treatment
\end{tabular}

If the subject used a rescue medication prior to one of the 1-,2- or 24-hour time points, the last pain score prior to the rescue medication was carried forward. If rescue therapy was used before the 1-hour assessment, the baseline VAS score was carried forward.

Secondary outcomes were the mean change in baseline pain VAS scores at 2 hours after starting treatment (if a rescue medication was not used); mean change in baseline pain VAS score at 24 hours after starting treatment (if a rescue medication was not used); the proportion of subjects not requiring a rescue medication at 2 hours; the proportion of subjects not requiring a rescue medication within 24 hours of starting treatment; the proportion of subjects who were pain-free at 1,2 and 24 hours after starting treatment (if a rescue medication was not used); the proportion of treatment responders, defined as subjects with $\mathrm{a} \geq 50 \%$ decrease in baseline pain VAS score at 1,2 and 24 hours after starting treatment (if a rescue medication was not used). An additional ad hoc analysis was headache relief, defined as improvement from baseline moderate or severe pain to mild or no pain. Standard pain categories were derived based on VAS scores: (0-1, No Pain; 2-3, Mild Pain; 4-6, Moderate Pain. 7-10, Severe Pain) (31-33).

\section{Statistical Analysis}


Statistical analyses were performed on the intent-to-treat (ITT) population, comprised of all randomized subjects, and on the modified intent-to-treat (mITT) population. The mITT set included all randomized subjects who completed Visit 1, received the device for home use, had baseline and 1-hour VAS data reported not out of window, a baseline VAS score of $\geq 2$, and achieved a minimal effective level of stimulation per the protocol. If a rescue medication was used, the previous VAS score was carried forward. The ITT analysis served as the main analysis set for all safety evaluations and the MITT set served for efficacy analysis (active $v s$. sham stimulation). The time post-treatment initiation ( $t_{0}$, baseline; $t_{1}, 1$ hour (end of treatment); $t_{2}, 2$ hours; $t_{3}, 24$ hours) and group (sham $v s$. active stimulation) were independent factors.

Data were summarized with descriptive statistics and are presented in tables and figures. Continuous variables were summarized by a mean, standard deviation, minimum, median and maximum, and categorical variables by count and percentage. For comparison of means (continuous variables), the twosample t-test or the Wilcoxon rank sum test were used, as appropriate. The percentage decrease from baseline of the VAS was analyzed with repeated measures ANCOVA. The decrease was modelled as a function of group, time-points (entered as categorical variable) and the group by time interaction term, baseline VAS score was entered as a covariate. For comparison of proportions (categorical variables), the chi-squared test or Fisher's exact test were used, as appropriate. The overall significance level for this study was $5 \%$ using two-tailed tests. Secondary endpoints were exploratory in nature, therefore, per secondary end points, nominal p-values are presented. Where confidence limits are appropriate, the confidence level was set at $95 \%$. Data were analyzed using commercial statistical software (SAS ${ }^{\circledR}$ version 9.4. SAS Institute, Inc.; Cary, NC).

\section{Results}

This study was conducted between February 8, 2018, and November 11, 2018. The mean (SD) age of enrolled subjects was 30 (8.34) years (range, $20-58$ years) and most $(n=45,82 \%)$ were female. The mean age of migraine onset was 16.2 (4.7) years (range, 4 to 30 years). The mean minimum and maximum monthly migraine days were $3.8(2.8)$ and $4.6(3.1)$, respectively. Most subjects $(n=33 ; 60 \%)$ had migraine without aura and few $(n=4,7.3 \%)$ had chronic migraine. There were no significant group differences in demographics or baseline characteristics. The demographics of study subjects are summarized in Table 1 and their migraine history is summarized in Table 2. 
Table 1

Demographics and Baseline Characteristics of Enrolled Subjects

\begin{tabular}{|lll|}
\hline & $\begin{array}{l}\text { Treatment } \\
\mathbf{n = 2 7}\end{array}$ & $\begin{array}{l}\text { Sham } \\
\mathbf{n = 2 8}\end{array}$ \\
\hline Mean Age (SD), years & $29.2(8.6)$ & $30.8(8.2)$ \\
\hline Median Age (range), years & $26.8(20.2,57.6)$ & $28.7(21.0,47.6)$ \\
\hline Gender, \% & & \\
\hline Male & 18.5 & 17.9 \\
\hline Female & 81.5 & 82.1 \\
\hline Mean BMI (SD), kg/m² & $24.8(4.2)$ & $24.2(4.9)$ \\
\hline Median BMI (range), kg/m ${ }^{2}$ & $25.2(19.0,40.1)$ & $24.0(17.7,39.3)$ \\
\hline Mean Head Circumference (SD), cm & $55.4(2.1)$ & $54.6(2.5)$ \\
\hline Median Head Circumference (range), cm & $55.0(51,59)$ & $54.5(51,59)$ \\
\hline BMl, body-mass index; SD, standard deviation. & \\
\hline
\end{tabular}


Table 2

Migraine History Among Enrolled Subjects

\begin{tabular}{|llll|}
\hline & Total & Treatment & Sham \\
& $\mathbf{N = 5 5}$ & $\mathbf{n = 2 7}$ & $\mathbf{n = 2 8}$ \\
\hline Mean Age of migraine Onset (SD), years & $16.2(4.70)$ & $15.4(4.44)$ & $17.0(4.90)$ \\
\hline Median Age of migraine Onset (range), years & $16.0(4,30)$ & $15.0(6,26)$ & $18.0(4,30)$ \\
\hline Mean Minimum Monthly Migraine Attacks (SD) & $3.8(2.84)$ & $3.7(2.66)$ & $3.9(3.04)$ \\
\hline Median Minimum Monthly Migraine Attacks (range) & $4.0(1,12)$ & $4.0(1,12)$ & $3.0(1,12)$ \\
\hline Mean Maximum Monthly Migraine Attacks (SD) & $4.6(3.11)$ & $4.4(3.17)$ & $4.8(3.10)$ \\
\hline Median Maximum Monthly Migraine Attacks (range) & $4.0(1,12)$ & $4.0(1,12)$ & $4.0(2,12)$ \\
\hline Mean Minimum Monthly Headache Days (SD) & $5.0(4.81)$ & $4.8(4.57)$ & $5.2(5.11)$ \\
\hline Median Minimum Monthly Headache Days (range) & $4.0(1,27)$ & $4.0(1,24)$ & $4.0(1,27)$ \\
\hline Mean Maximum Monthly Headache Days (SD) & $7.0(5.32)$ & $6.4(4.97)$ & $7.6(5.67)$ \\
\hline Median Maximum Monthly Headache Days (range) & $5.0(1,27)$ & $5.0(1,25)$ & $5.5(2,27)$ \\
\hline Mean Pain Locations (SD) & $\mathrm{N}(\%)$ & $\mathbf{n}(\%)$ & $\mathbf{n}(\%)$ \\
\hline Front right & $47(85.5)$ & $22(81.5)$ & $25(89.3)$ \\
\hline Front left & $47(85.5)$ & $25(92.6)$ & $22(78.6)$ \\
\hline Rear right & $30(54.5)$ & $12(44.4)$ & $18(64.3)$ \\
\hline Rear left & $30(54.5)$ & $15(55.6)$ & $15(53.6)$ \\
\hline Mean Migraine Without Aura (SD) & $33(60.0)$ & $18(66.7)$ & $15(53.6)$ \\
\hline Mean Migraine With Aura (SD) & $18(32.7)$ & $7(25.9)$ & $11(39.3)$ \\
\hline Mean Chronic Migraine (SD) & $4(7.3)$ & $2(7.4)$ & $2(7.1)$ \\
\hline SD, standard deviation. & & & \\
\hline
\end{tabular}

\section{Efficacy Outcomes}

Baseline pain scores on the continuous (VAS) and categorical scale are shown in Table 3. There were no significant group differences in baseline pain VAS scores or migraine history. The between-group difference in percentage decrease in baseline VAS scores were significant at all time points with the decrease significantly greater in the active treatment group. The group difference at 1 hour was $42.8 \%$ $(95 \% \mathrm{Cl} 20.57 \%, 62.18 \% ; p=0.0002)$, at 2 hours was $34.7 \%(95 \% \mathrm{Cl} 2.88 \%, 62.80 \% ; p=0.0324)$ and at 24 hours was $37.0 \%(95 \% \mathrm{Cl} 5.46 \%, 66.96 \% ; p=0.0220)$. The change in mean pain intensity VAS scores is summarized in Table 4 and the percent reduction in mean baseline VAS scores are shown in Fig. 3. 
Table 3

Baseline Visual Analog Pain Scores, Modified Intent-to-

Treat Population

\begin{tabular}{|lll|}
\hline & $\begin{array}{l}\text { Treatment } \\
\mathbf{n = 2 5}\end{array}$ & $\begin{array}{l}\text { Sham } \\
\mathbf{n = 2 6}\end{array}$ \\
\hline Mean Score (SD) & $5.7(1.97)$ & $5.4(2.06)$ \\
\hline Median Score (range) & $5.0(2.0,9.0)$ & $6.0(2.0,9.0)$ \\
\hline Pain Level, n (\%) & & \\
\hline Mild & $4(16.0)$ & $6(23.1)$ \\
\hline Moderate & $11(44.0)$ & $13(50.0)$ \\
\hline Severe & $10(40.0)$ & $7(26.9)$ \\
\hline SD, standard deviation. & & \\
\hline
\end{tabular}

Table 4

Change in Visual Analog Scale Pain Scores Over Time, Modified Intent-to-Treat Population

\begin{tabular}{|lllll|}
\hline & \multicolumn{2}{c}{ Treatment } & Sham & \\
\cline { 2 - 5 } & Mean (SD) & Median (range) & Mean (SD) & Median (range) \\
\hline Time (hours) & & & & \\
\hline 0 (Baseline), $\mathrm{n}=25$ & $5.7(2.0)$ & $5.0(2.0,9.0)$ & $5.4(2.06)$ & $6.0(2.0,9.0)$ \\
\hline $1, \mathrm{n}=24$ & $2.6(1.7)$ & $2.0(0.0,7.0)$ & $4.8(2.69)$ & $6.0(0.0,9.0)$ \\
\hline $2, \mathrm{n}=24$ & $2.5(2.1)$ & $2.5(0.0,7.0)$ & $4.5(3.07)$ & $5.0(0.0,10.0)$ \\
\hline $24, \mathrm{n}=23$ & $1.6(2.3$ & $0.0(0.0,7.0)$ & $3.4(3.40)$ & $2.0(0.0,10.0)$ \\
\hline SD, standard deviation. & & & \\
\hline
\end{tabular}

The proportion of pain-free subjects was numerically higher in the active treatment group at 1 hour (29.2\% vs. $16.0 \%), 2$ hours ( $41.7 \%$ vs. $20.0 \%$ ) and 24 -hours (65\% vs. $40.0 \%)$.

Although the between-group difference was not statistically significant, the number of pain-free subjects was more than twice as great among subjects in the active treatment group at 2 hours (Table 5a, Fig. 4A). The pain freedom between-group difference 2-hours post treatment among subjects with severe or moderate baseline pain, was significantly higher in the active treatment group (42.86\% vs. $10.53 \%, p=$ 0.02; Table 5b, Fig. 4B). 
Table 5

a. Pain-Free Subjects versus Baseline, Modified Intent-to-Treat Population

Treatment

Time (hours)

1

2

24

Table 5b. Pain-Free Subjects with Severe or Moderate Pain at Baseline, Modified Intent-to-Treat Population

Treatment

Time (hours)

1

$2^{a}$

24 $\mathrm{n} / \mathrm{N}(\%)$

$7 / 24(29.2)$

10/24 (41.7)

$15 / 23(65.2)$
Sham

$\mathrm{n} / \mathrm{N}(\%)$

$4 / 25(16.0)$

$5 / 25(20.0)$

$10 / 25(40.0)$

\begin{tabular}{lll} 
& Treatment & Sham \\
Time (hours) & $\mathrm{n} / \mathrm{N}(\%)$ & $\mathrm{n} / \mathrm{N}(\%)$ \\
\hline 1 & $5 / 21(23.8)$ & $2 / 19(10.5)$ \\
$2^{\mathrm{a}}$ & $9 / 21(42.9)$ & $2 / 19(10.5)$ \\
24 & $12 / 20(60.0)$ & $7 / 19(36.8)$
\end{tabular}

${ }^{\mathrm{a}} p=0.0217$, Fisher's exact test.

Responder rates of subjects achieving $\geq 50 \%$ improvement of baseline VAS scores were significantly higher in the active treatment group at 1 hour $(66.7 \%$ vs. $20.0 \% ; p=0.0014), 2$ hours $(66.7 \% v s .32 .0 \% ; p=$ $0.0227)$ and 24 hours $(78.3 \%$ vs. $48.0 \% ; p=0.0401)$. Responder rate results are presented in Fig. 5.

Headache relief, defined as improvement from severe or moderate baseline pain to mild or no pain, was significantly greater among active treatment subjects at 1 hour $(66.7 \% v s .26 .3 \% ; p=0.0140)$ and 2 hours (76.2\% vs. 31.6\%; $p=0.0100)$ and approached significance at 24 hours $(80 \% v s .52 .6 \% ; p=0.0958)$. Headache relief results are presented in Fig. 6.

Adverse events (AEs) were reported by 14 subjects in the active treatment group $(n=6)$ and sham group $(n=8)$. There was no statistically significant difference between the groups in incidence of AEs. Seven reported AEs among subjects receiving active treatment were headache $(n=2)$, numbness/ paresthesia $(n=2)$, skin irritation $(n=1)$, nausea $(n=1)$ and itching scalp $(n=1)$. All were mild in severity except one reported headache which was of moderate severity and considered as possibly related to the device. All events resolved spontaneously. Eight reported AEs among sham-treated subjects were nausea $(n=3)$, vomiting $(n=2)$, tiredness $(n=1)$, palpitation $(n=1)$, pressure in the head $(n=1)$, and photophobia $(n=1)$. All were mild in severity except one report of vomiting which was of moderate severity. Tiredness and palpitation were considered to be possibly treatment-related. All AEs resolved spontaneously.

\section{Discussion}


The objective of this study was to determine if a self-administered stimulation protocol using the eCOTNS device can provide a safe, effective, and fast-acting reduction of migraine-related pain. As hypothesized, the device was shown to be significantly effective across multiple clinically relevant outcome measures. At 1 hour post-treatment, the active stimulation group showed a mean $53.1 \%$ reduction in pain severity compared to a mean $10.3 \%$ decrease among sham-treated subjects, a difference of $42.8 \%$. Pain reduction was maintained at 2 hours with $52 \%$ and $17.3 \%$ decreases in pain severity, respectively, a difference of $34.7 \%$. The treatment effect is also durable, as the proportion of pain reduction among active treatment subjects was $71.3 \%$ at 24 hours vs. $34.3 \%$ for the sham group. The responder rate was also significantly higher in the active treatment group at all time points and the proportion of pain-free subjects at 2 hours post treatment was more than two-fold higher in the active group $(41.7 \%$ vs. $20 \%)$. Although not statistically significant, it suggests a clinically meaningful response. At a post hoc pain-free assessment analysis of a subgroup of subjects with moderate or severe baseline headache pain, the between-group difference was in favor of the active group again at 1 and 2 hours. These results support the safety and efficacy of the eCOT-NS, eventually leading to its clearance of for the acute treatment of migraine,

These clinical results were substantially higher in aborting migraine when compared to a 60-minute session of single channel non-invasive trigeminal neurostimulation (16). In that study, treated subjects achieved a net response rate of $29 \%, 18 \%$ and $17 \%$ at 1,2 and 24 hours, respectively, compared to a net response of $42.8 \%, 34.7 \%$, and $37 \%$ in the present study with eCOT-NS. In another study, subjects implanted with an occipital nerve stimulator achieved a responder rate defined as headache days per month of $39 \%$ vs. $6 \%$ among sham-treated subjects, a net difference of $33 \%$. Subjects receiving active stimulation in the current study achieved a responder rate, defined as a $50 \%$ reduction in pain intensity, of $66.7 \%$ vs. $20.0 \%$ among sham-treated subjects ay 1 hour post-treatment, a difference of $46.7 \%$. Comparing the result of the current study with other clinical trials should be done with caution as the selection criteria and outcome measures may vary, nevertheless, the results of the current study support the efficacy of combined stimulation of both the trigeminal and occipital nerves for the acute treatment of migraine.

Enrolled subjects in this trial had one to six monthly migraine episodes similar to subjects enrolled in large-scale triptan trials (34) and are representative of migraine patients in the general population in need of migraine-abortive treatment (2). The results achieved with eCOT-NS appear to be comparable to oral triptans, as demonstrated by $76 \%$ headache relief 2 hours post treatment in our study, compared to $42-$ $76 \%$ reported following oral triptans (34). Recent studies of new medications for the acute treatment of migraine, including ubrogepant (35) and rimegepant (36), appear to have more modest pain relief efficacy compared to eCOT-NS ( $60.7 \%$ and $59.3 \%$ at 2 hours, respectively).

An additional benefit of the Relivion $® M G$ system is that it utilizes a custom mobile application designed for continuously recording data and securely uploading it to a cloud database to enable remote treatment monitoring by the patient physician. Treatment reports are available to the treating physicians over a webbased interface. By remote monitoring, physicians can assess patient adherence to self-administered 
treatments and success of aborting or minimizing migraine severity. Using the aggregated data presented on the physician interface, the physician can optimize treatment by recommending treatment regimen adjustments, such as adjusting the stimulation time and intensity.

Treatment with eCOT-NS provided clinical benefits for migraine subjects with an acceptable safety profile. The reported AEs associated with the eCOT-NS device were relatively few, of mild severity and resolved spontaneously with no intervention. This compares favorably with surgical risks associated with electrode implants or systemic AEs associated with drug administration (2). The use of eCOT-NS might enable migraine patients to discontinue current migraine medications.

Limitations of the study include the modest number of subjects which did not permit subgroup analysis based on headache severity. Another shortcoming was the efficacy assessment based on a single 60minute stimulation session which limited assessment of long-term efficacy.

\section{Conclusions}

These results suggests that many migraine patients achieve clinically significant pain relief and pain freedom with eCOT-NS, a non-invasive, home-based, self-administered, neuromodulation of the trigeminal-occipital neural network. These beneficial effects appear to demonstrate comparable or higher efficacy compared to existing migraine treatments, without the adverse effects associated with medications or the surgical risks of invasive electrode procedures. eCOT-NS, with its physician unique remote monitoring capabilities, may optimize migraine treatment care and patient adherence and by that migraine patient's quality of life.

\section{Abbreviations}

\begin{tabular}{ll} 
BMI & Body-mass index \\
\hline eCOT-NS & external concurrent occipital and trigeminal neurostimulation \\
\hline PNS & peripheral nerve stimulation \\
\hline TCC & trigeminocervical complex \\
\hline SD & Standard deviation \\
\hline VAS & visual analogue scale
\end{tabular}

\section{Declarations}

\section{Ethics approval and consent to participate}

This study was approved by the Institution Ethics Committee of the Laniado Medical Center, Netanya, Israel. Enrolled subjects provided written informed consent prior to participating in any study-related activities. 


\section{Consent for publication}

Not applicable.

\section{Availability of data and materials}

The study description can be found on ClinicalTrials.gov, Identifier NCT03398668.

Available: https://clinicaltrials.gov/ct2/show/NCT03398668. All data generated or analyzed during this study are included in this published article.

\section{Competing interests}

RS and ST are advisors to Neurolief Ltd. The other authors declare they have nothing to disclose. LD Works as a biostatistician for hire and receives financial compensation for performing statistical analyses for companies in the medical device industry. There are no other conflicts of interest.

\section{Funding}

Neuroleif, Ltd. provided funding for support editorial assistance for this manuscript.

\section{Authors' contributions}

DO was the study principal investigator and enrolled all subjects, LD performed the study statistical design and performed all statistical analysis, ST consulted during study design and manuscript preparation and RS acted as medical advisor and reviewed all adverse events.

\section{Acknowledgements}

Editorial assistance was provided by Carl S. Hornfeldt, PhD, Apothekon. Inc., during the preparation of this manuscript.

\section{References}

1. Migraine Research Foundation 2021. Available: https://migraineresearchfoundation.org/aboutmigraine/migraine-facts/. Accessed: December 20, 2021.

2. Lipton RB, Bigal ME, Diamond M, et al. Migraine prevalence, disease burden, and the need for preventive therapy. Neurology 2007; 8:343-49.

3. Pescador Ruschel MA, De Jesus O. Migraine Headache. Treasure Island, FL: StatPearls Publishing; 2021.

4. Mayans $L$, Walling A. Acute migraine headache: treatment strategies. Am Fam Physician 2018;97:243-51.

5. Jamieson DG. The safety of triptans in the treatment of patients with migraine. Am J Med 2002;112:135-40. 
6. Tepper SJ, Millson D. Safety profile of the triptans. Expert Opin Drug Saf 2003;2:123-32.

7. Loder E. Triptan therapy in migraine. N Engl J Med 2010;363:63-70.

8. Robbins MS, Lipton RB. Transcutaneous and percutaneous neurostimulation for headache disorders. Headache 2016;57:4-13.

9. Miller S, Watkins L, Matharu M. Long-term outcomes of occipital nerve stimulation for chronic migraine: a cohort of 53 patients. J Headache Pain 2016;17:68.

10. Palmisani S, Al-Kaisy A, Arcioni R, et al. A six year retrospective review of occipital nerve stimulation practice-controversies and challenges of an emerging technique for treating refractory headache syndromes. J Headache Pain 2013;14:67.

11. Silberstein SD, Dodick DW, Saper J, et al. Safety and efficacy of peripheral nerve stimulation of the occipital nerves for the management of chronic migraine: results from a randomized, multicenter, double-blinded, controlled study. Cephalalgia 2012;32:1165-79.

12. Serra G, Marchioretto F. Occipital nerve stimulation for chronic migraine: a randomized trial. Pain Physician 2012;15.

13. Saper JR, Dodick DW, Silberstein SD, et al. Occipital nerve stimulation for the treatment of intractable chronic migraine headache: ONSTIM feasibility study. Cephalalgia 2011;31:271-85.

14. Dodick DW, Silberstein SD, Reed KL, et al. Safety and efficacy of peripheral nerve stimulation of the occipital nerves for the management of chronic migraine: long-term results from a randomized, multicenter, double-blinded, controlled study. Cephalalgi 2015;35:344-58.

15. Schoenen J, Vandersmissen B, Jeangette S, et al. Migraine prevention with a supraorbital transcutaneous stimulator: a randomized controlled trial. Neurology 2013;80:697-704.

16. Chou DE, Shnayderman Yugrakh $M$, Winegarner $D$, et al. Acute migraine therapy with external trigeminal neurostimulation (ACME): A randomized controlled trial. Cephalalgia 2019;39:3-14.

17. Magis D, Sava S, d'Elia TS, et al. Safety and patients' satisfaction of transcutaneous supraorbital neurostimulation (tSNS) with the Cefaly ${ }^{\circledR}$ device in headache treatment: a survey of 2,313 headache sufferers in the general population. J Headache Pain 2013;14:95.

18. Ashkan K, Sokratous G, Göbel H, et al. Peripheral nerve stimulation registry for intractable migraine headache (RELIEF): a real-life perspective on the utility of occipital nerve stimulation for chronic migraine. Acta Neurochir (Wien) 2020;162:3201-11.

19. Reed KL, Will KR, Conidi F, et al. Concordant occipital and supraorbitaln eurostimulation therapy for hemiplegic migraine; initial experience; a case series. Neuromodulation 2015;18:297-304.

20. Hann S, Sharan A. Dual occipital and supraorbital nerve stimulation for chronic migraine: a singlecenter experience, review of literature, and surgical considerations. Neurosurg Focus 2013;35:E9.

21. Reed KL, Black SB, Banta CJ, et al. Combined occipital and supraorbital neurostimulation for the treatment of chronic migraine headaches: initial experience. Cephalalgia 2010;30:260-71.

22. Reed KL. Peripheral neuromodulation and headaches: history, clinical approach, and considerations on underlying mechanisms. Curr Pain Headache Rep 2013;17:305. 
23. Reed KL, Will KR, Chapman J, et al. Combined occipital and supraorbital neurostimulation for chronic migraine headaches: an extended case series. Cephalalgia 2011;31:98.

24. Jiang JF, Diaz AN, Campbell M, et al. Supraorbital occipital circumferential stimulation (SOCS) for the treatment of refractory chronic primary headache: a case series. World Neurosurg 2019;\$18788750(18)32931-0.

25. Ahmed HE, White PF, Craig WF, et al. Headache. 40 2000:311-15.

26. Mueller O, Hagel V, Wrede $K$, et al. Stimulation of the greater occipital nerve: anatomical considerations and clinical implications. Pain Physician 2014;16:E181-9.

27. Tubbs RS, Salter EG, Wellons JC, et al. Landmarks for the identification of the cutaneous nerves of the occiput and nuchal regions. Clin Anat 2007;20:235-38.

28. General Assembly of the World Medical Association. World Medical Association Declaration of Helsinki: ethical principles for medical research involving human subjects. J Am Coll Dent 2014;81:14-8.

29. Headache Classification Committee of the International Headache Society (IHS). The International Classification of Headache Disorders, 3rd edition (beta version). Cephalalgia 2013;33:629-808.

30. Oved D, Sharon R, Tepper SJ. A device review of Relivion ${ }^{\circledR}$ : an external combined occipital and trigeminal neurostimulation (eCOT-NS) system for self-administered treatment of migraine and major depressive disorder. Expert Rev Med Devices 2021;18:333-42.

31. Yarnitsky D, Dodick DW, Grosberg BM, et al. Remote electrical neuromodulation (REN) relieves acute migraine: a randomized, double-blind, placebo-controlled, multicenter trial. Headache 2019;59:124052.

32. Rapoport AM, Bonner JH, Lin T, et al. Remote electrical neuromodulation (REN) in the acute treatment of migraine: a comparison with usual care and acute migraine medications. J Headache Pain 2019;20:83.

33. Yarnitsky D, Volokh L, Ironi A, et al. Nonpainful remote electrical stimulation alleviates episodic migraine pain. Neurology 2017;88:1250-55.

34. Cameron C, Kelly S, Hsieh SC, et al. Triptans in the acute treatment of migraine: a systematic review and network meta-analysis. Headache 2015;55:221-35.

35. Dodick DW, Lipton RB, Ailani J, et al. Ubrogepant for the treatment of migraine. N Engl J Med 2019;381:2230-41.

36. Croop R, Goadsby PJ, Stock DA, et al. Efficacy, safety, and tolerability of rimegepant orally disintegrating tablet for the acute treatment of migraine: a randomised, phase 3 , double-blind, placebo-controlled trial. Lancet 2019;394:737-45.

\section{Figures}




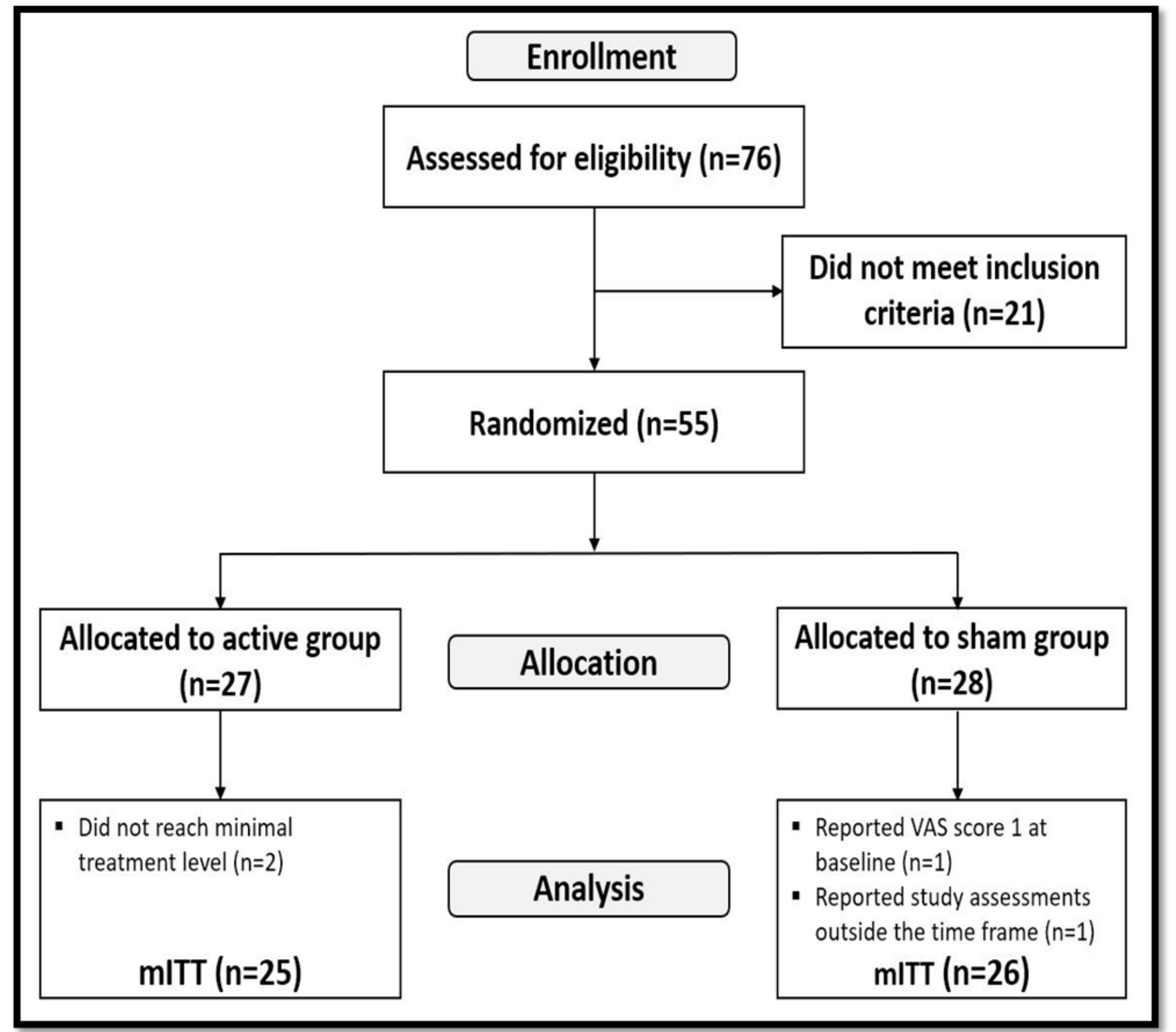

Figure 1

Consort Flow Diagram

Among the randomized subjects $(N=55)$, the subjects completing the study in the active treatment group $(n=25)$ and sham group $(n=26)$ formed the modified intent-to-treat population (mITT). 


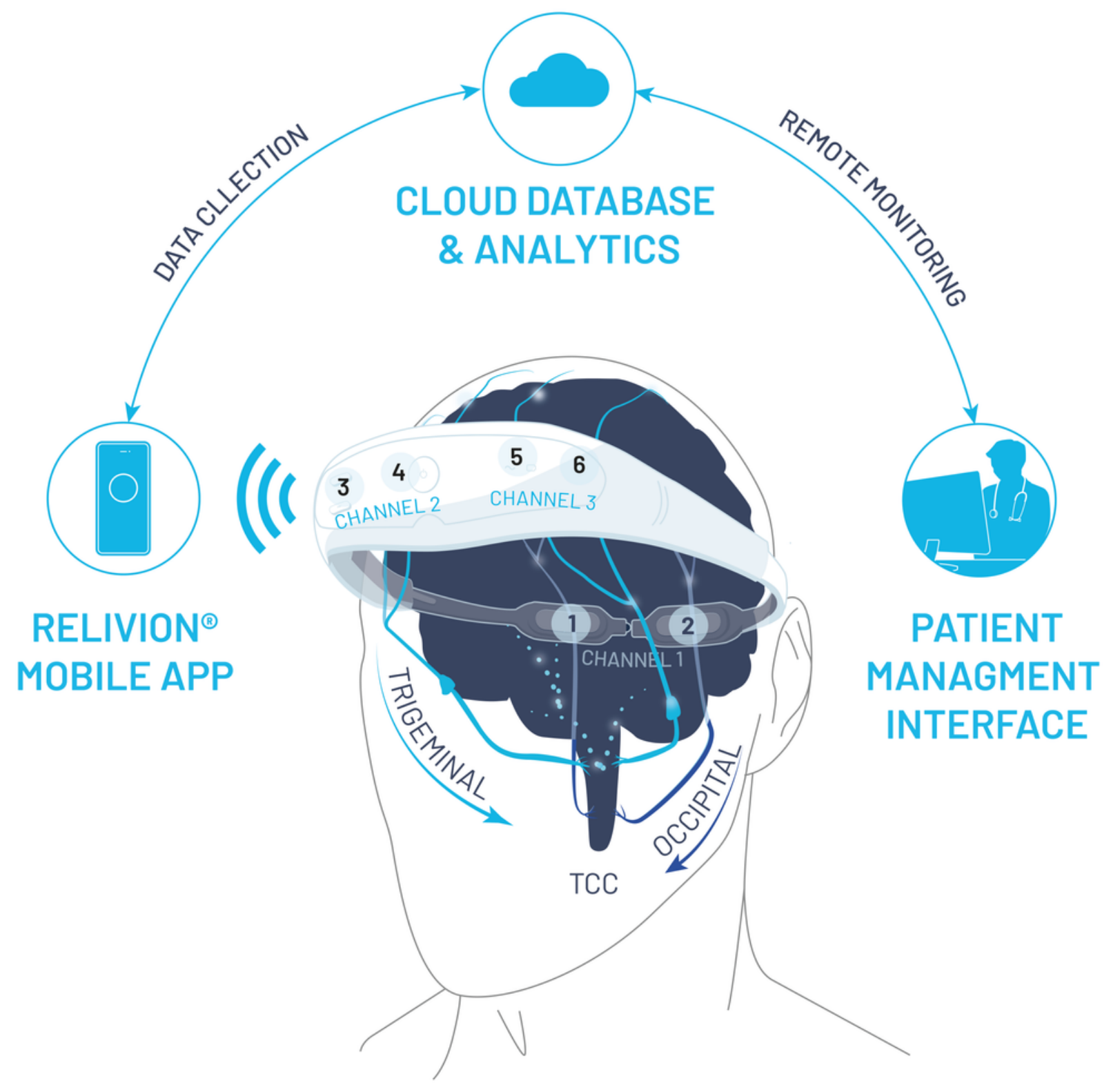

Figure 2

\section{The Relivion ${ }^{\circledR}$ MG System}

The eCOT-NS device provides noninvasive neuromodulation by concurrently stimulating the two primary nerve pathways in the brain associated with migraine, precisely targeting six nerve branches across the occipital and trigeminal nerves. The device is connected to a custom mobile application designed to 
upload treatment data to a cloud database. Treatment reports and metrices are available to the investigator over a web-based physician interface.

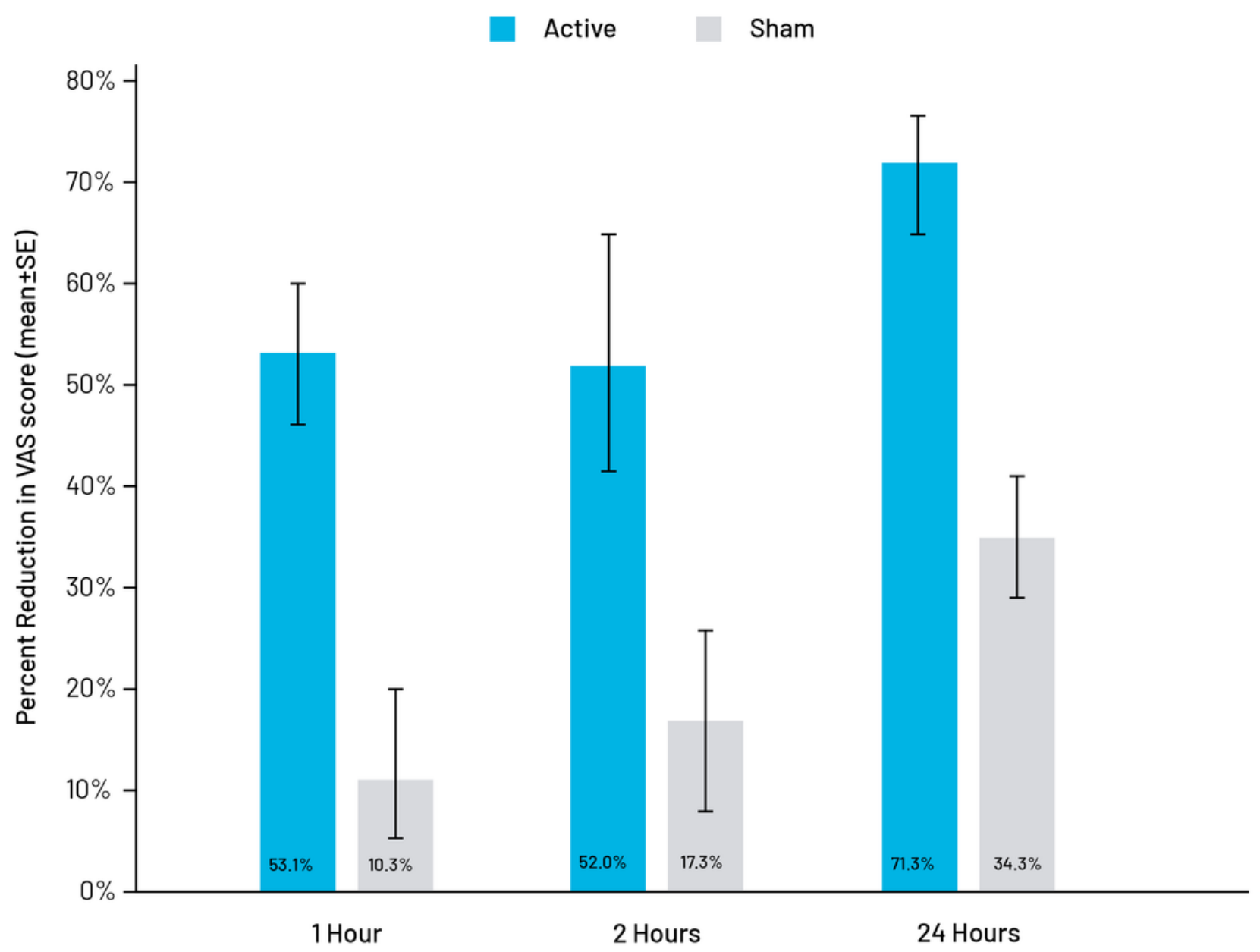

Figure 3

\section{Change in Percent Pain Intensity}

The therapeutic gain in pain intensity based on visual analog scale (VAS) scores was $42.8 \%$ at 1 hour post-treatment ( $p=0.0002), 34.7 \%$ at 2 hours $(p=0.0324)$ and $37.0 \%$ at 24 hours $(p=0.0220)$. 


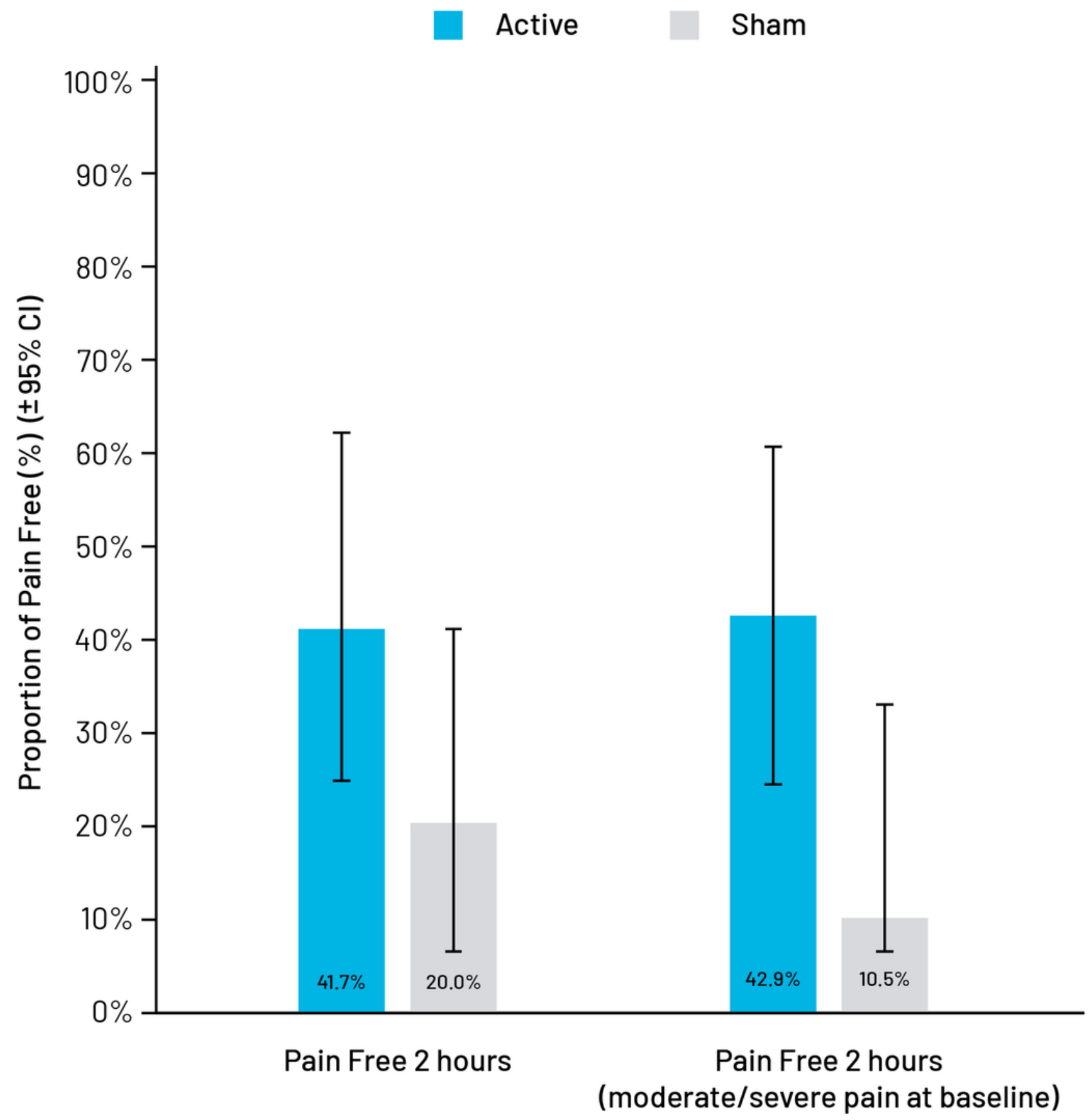

Figure 4

\section{Proportion of Pain-Free Subjects, Modified Intent-to-Treat Population}

The proportion of subjects who were pain-free at 1,2 and 24 hours without requiring rescue medications was greater in the active stimulation group. Overall, the proportion of pain-free subjects was numerically superior rate in the active treatment group at 2 hours (41.7\% vs. 20.0\%) (4A). Among subjects with baseline moderate or severe pain, the difference was even greater ( $42.9 \%$ vs. $10.5 \%, p=0.0217)(4 B)$. 


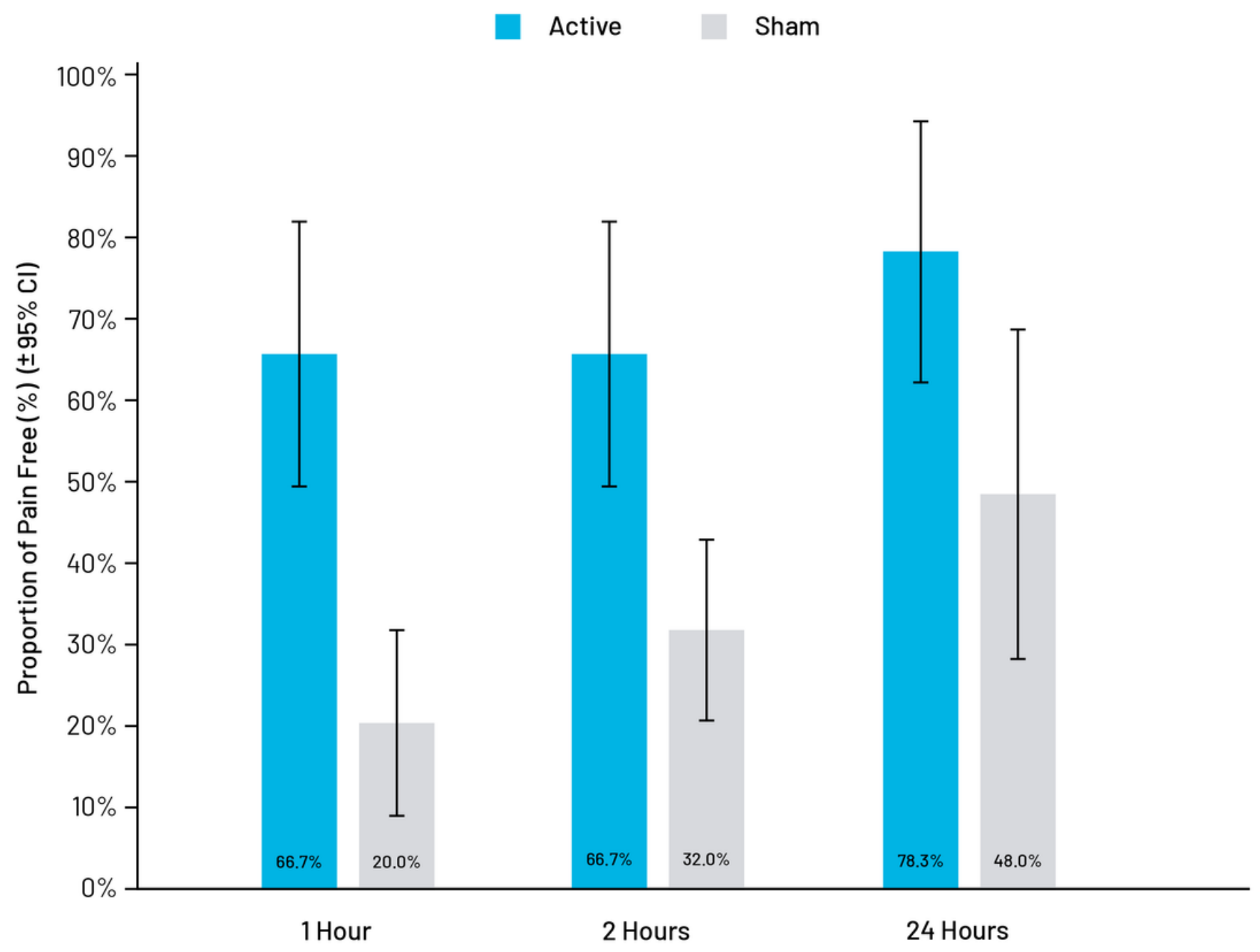

Figure 5

Proportion of Subjects Responding to Treatment, Modified Intent-to-Treat Population

The number of subjects achieving $\geq 50 \%$ improvement of baseline VAS pain scores (responders) was significantly higher in the active treatment group at 1 hour $(66.7 \%$ vs. $20.0 \% ; p=0.0014), 2$ hours $(66.7 \%$ vs. $32.0 \% ; p=0.0227)$ and 24 hours $(78.3 \%$ vs. $48.0 \% ; p=0.0401)$. 


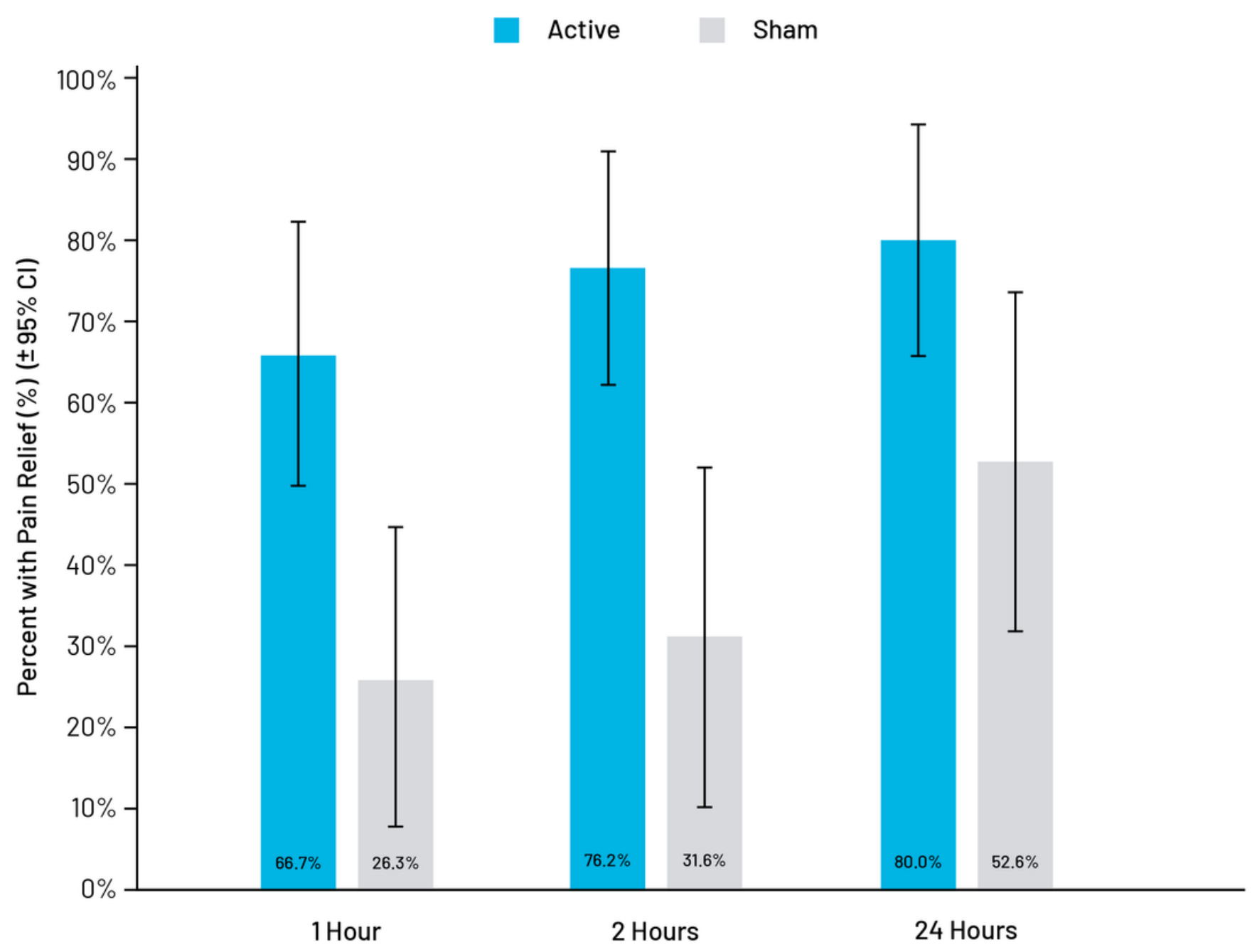

Figure 6

\section{Subjects with Headache Relief Following Treatment}

The proportion of subjects experiencing Headache Relief, defined as improvement from severe or moderate baseline pain to mild or no pain, was significantly greater among active treatment subjects at 1 hour $(66.7 \%$ vs. $26.3 \% ; p=0.0140)$ and 2 hours $(76.2 \%$ vs. $31.6 \% ; p=0.0100)$ and approached significance at 24 hours $(80 \% v s .52 .6 \% ; p=0.0958)$. 